\title{
Haar Wavelet Transform Based Facial Emotion Recognition
}

\author{
Siyuan Lu, a and Fidel Evans ${ }^{23, b}$ \\ ${ }^{1}$ School of Computer Science \& Technology, Nanjing Normal University, Nanjing, 210023, China \\ ${ }^{2}$ Herbert Wertheim College of Engineering, University of Florida, Gainesville, FL 32611, USA \\ ${ }^{3}$ School of Information, Florida State University, Tallahassee, FL 32304, USA \\ a269125022@qq.com, bf.evans.uf@gmail.com
}

Keywords: Haar wavelet transform; Emotion recognition; Facial expression

\begin{abstract}
In order to propose an effective emotion recognition system, this paper proposed a novel Haar wavelet transform based emotion recognition system. This system is based on facial expression images obtained by digital camera. Our method used principal component analysis as the dimensionality reduction approach. The support vector machine (SVM) was used as the classifier. The results showed that our method achieved an accuracy of $78.37 \pm 1.50 \%$. In all, our method is effective in emotion recognition.
\end{abstract}

\section{Background}

Emotion recognition (ER) identifies the motion of humans. The typical methods are based on facial expression images obtained by digital camera. Traditional ER methods are usually developed a three-stage algorithm: feature extraction, feature reduction, and classification. Besides, ER can be applied to detect neurological and psychiatric diseases.

In the last decade, many new methods were proposed. Drume (2012) [1] proposed a multi-level classifier (MLC). Lu (2016) [2] used biorthogonal wavelet entropy (BWE). In the classification stage, they used fuzzy support vector machine. Yogesh (2017) [3] used a hybrid particle swarm optimization assisted biogeography-based optimization.

In this study, we proposed a novel method based on Haar wavelet transform (HWT), principal component analysis (PCA), and support vector machine (SVM). These three traditional components help the success of our system.

\section{Materials and Methods}

The 42 volunteers were enrolled by advertisements. These volunteers lay seven facial expressions (happy, sadness, surprise, anger, disgust, fear, and neutral). Each volunteer showed five different pictures for each emotion, and thus generated in total 35 pictures. For the 42 volunteers, we generated 1470 images. Then, we extract the face region by Photoshop.

The Haar wavelet is a square-shaped functions, which generate a wavelet family. Haar wavelet is the most famous wavelet in the field of discrete wavelet transform [4-9]. Its mother wavelet $\delta(a)$ is defined as:

$$
\delta(a)=\left\{\begin{array}{cc}
+1 & 0 \leq a \leq 1 / 2 \\
-1 & 1 / 2 \leq a \leq 1 \\
0 & \text { otherwise }
\end{array}\right.
$$

Here a denotes the time domain. The scaling function $\sigma(a)$ is defined as:

$$
\sigma(a)=\left\{\begin{array}{cc}
1 & 0 \leq a<1 \\
0 & \text { otherwise }
\end{array}\right.
$$


In this study, we chose to use a three-level Haar wavelet transform.

Afterwards, the principal component analysis (PCA) was used to extract reduced features of at least $98 \%$ of the whole variance of original wavelet subband coefficients. The PCA can be regarded as a transformation that the first PC has largest variance, and the following PCs has largest variance and should be orthogonal to preceding PCs [10-15].

In the final stage, we used support vector machine (SVM) as the classifier. The SVM can analyze and classify data into two categories [16-21]. The SVM can generate a pair of hyperplanes, which separate the data of two different classes. Compared to feed-forward neural network [22-27] and multilayer perceptron [28-30], SVM is easy to train and its results are stable. Finally, 10 times of 10 -fold cross validation was used to measure the classifier.

\section{Experiments, Results, and Discussions}

The sensitivity results of the seven classes by our HWT + PCA + SVM are shown below in Table 1. The overall accuracy result is listed in Table 2. In all, our method achieves an overall accuracy (OA) of $78.37 \pm 1.50 \%$.

Table 1 Sensitivity result over 10 runs of 10 -fold cross validation

\begin{tabular}{|l|l|l|l|l|l|l|l|}
\hline Run & \multicolumn{1}{|c|}{ Anger } & \multicolumn{1}{|c|}{ Disgust } & \multicolumn{1}{|c|}{ Fear } & \multicolumn{1}{c|}{ Happy } & \multicolumn{1}{c|}{ Neutral } & \multicolumn{1}{c|}{ Sadness } & Surprise \\
\hline R1 & 76.19 & 72.86 & 77.62 & 81.43 & 78.57 & 78.10 & 79.52 \\
\hline R2 & 78.10 & 79.52 & 80.48 & 81.43 & 79.05 & 77.62 & 78.57 \\
\hline R3 & 83.33 & 81.43 & 80.00 & 79.05 & 77.62 & 78.10 & 77.14 \\
\hline R4 & 82.86 & 78.10 & 77.14 & 77.14 & 79.05 & 80.00 & 77.14 \\
\hline R5 & 76.19 & 77.62 & 77.62 & 73.81 & 75.24 & 77.62 & 76.19 \\
\hline R6 & 73.33 & 72.38 & 73.81 & 77.14 & 75.24 & 81.43 & 75.71 \\
\hline R7 & 81.90 & 81.43 & 78.57 & 80.00 & 82.86 & 74.76 & 80.48 \\
\hline R8 & 79.05 & 84.76 & 79.05 & 80.48 & 79.05 & 77.14 & 80.95 \\
\hline R9 & 76.19 & 76.19 & 80.00 & 78.57 & 79.05 & 80.00 & 79.05 \\
\hline R10 & 75.24 & 80.48 & 80.00 & 74.76 & 77.14 & 80.48 & 78.10 \\
\hline Avr & $78.24 \pm 3.45$ & $78.48 \pm 3.90$ & $78.43 \pm 2.01$ & $78.38 \pm 2.65$ & $78.29 \pm 2.20$ & $78.52 \pm 1.96$ & $78.29 \pm 1.76$ \\
\hline
\end{tabular}

Table 2 Accuracy result over 10 runs of 10-fold cross validation

\begin{tabular}{|l|l|l|l|}
\hline Run & Accuracy & Run & Accuracy \\
\hline R1 & 77.76 & R6 & 75.58 \\
\hline R2 & 79.25 & R7 & 80.00 \\
\hline R3 & 79.52 & R8 & 80.07 \\
\hline R4 & 78.78 & R9 & 78.44 \\
\hline R5 & 76.33 & R10 & 78.03 \\
\hline Avr & $78.37 \pm 1.50$ \\
\hline
\end{tabular}

The shortcoming of this method is lack of comparison with state-of-the-art approaches. Besides, we shall try to test kernel SVM [31-33]. Besides, the extreme learning machine (ELM) [34, 35] shall be used as an alternative to our system. Furthermore, the meta-parameters in our system are assigned by experience. In the future, we shall try to use bioinspired algorithms (such as pattern search [36], ant colony algorithm [37], particle swarm optimization [38-40], biogeography-based optimization [41-43], firefly algorithm, artificial bee colony [44-46], artificial immune system, etc.) to seek their optimal values.

\section{Conclusion and Future Research}

In this paper, we proposed a novel ER method. Its accuracy achieved $78.37 \pm 1.50 \%$. In the future studies, we shall try to increase its identification performance. Besides, the seven emotions may not 
cover all the human emotions. Hence, we may try to develop new human emotions based on the slight motions of facial muscles.

\section{Acknowledgment}

This paper is supported by Research Innovation Program for College Graduates of Jiangsu Province (KYLX16_1292).

\section{References}

[1] Drume, D. and A.S. Jalal. A Multi-level Classification Approach for Facial Emotion Recognition. in International Conference on Computational Intelligence And Computing Research (ICCIC). 2012. Coimbatore, INDIA: IEEE. pp. 288-292

[2] Lu, H.M., Facial Emotion Recognition Based on Biorthogonal Wavelet Entropy, Fuzzy Support Vector Machine, and Stratified Cross Validation. IEEE Access, 2016. 4: pp. 8375-8385

[3] Yogesh, C.K., et al., A new hybrid PSO assisted biogeography-based optimization for emotion and stress recognition from speech signal. Expert Systems with Applications, 2017. 69: pp. $149-158$

[4] Li, Y. Detection of Dendritic Spines using Wavelet Packet Entropy and Fuzzy Support Vector Machine. CNS \& Neurological Disorders - Drug Targets, 2016. 15, DOI: 10.2174/1871527315666161111123638 (Online).

[5] Wang, S.-H. Single slice based detection for Alzheimer's disease via wavelet entropy and multilayer perceptron trained by biogeography-based optimization. Multimedia Tools and Applications, 2016, DOI: 10.1007/s11042-016-4222-4 (Online).

[6] Nayak, D.R. Detection of unilateral hearing loss by Stationary Wavelet Entropy. CNS \& Neurological Disorders - Drug Targets, 2017. 16, DOI: 10.2174/1871527315666161026115046 (Online).

[7] Gorriz, J.M. and J. Ramírez, Wavelet entropy and directed acyclic graph support vector machine for detection of patients with unilateral hearing loss in MRI scanning. Frontiers in Computational Neuroscience, 2016. 10, Article ID: 160

[8] Sun, P., Preliminary research on abnormal brain detection by wavelet-energy and quantum-behaved PSO. Technology and Health Care, 2016. 24(s2): pp. S641-S649

[9] Yang, J.Q., et al., A Novel Compressed Sensing Method for Magnetic Resonance Imaging: Exponential Wavelet Iterative Shrinkage-Thresholding Algorithm with Random Shift. International Journal Of Biomedical Imaging, 2016, Article ID: 9416435

[10]Chen, Y. and X.-Q. Chen Sensorineural hearing loss detection via discrete wavelet transform and principal component analysis combined with generalized eigenvalue proximal support vector machine and Tikhonov regularization. Multimedia Tools and Applications, 2016, DOI: 10.1007/s11042-016-4087-6 (Online).

[11]Zhan, T.M. and Y. Chen, Multiple Sclerosis Detection Based on Biorthogonal Wavelet Transform, RBF Kernel Principal Component Analysis, and Logistic Regression. IEEE Access, 2016. 4: pp. 7567-7576

[12] Wu, L., An MR brain images classifier via principal component analysis and kernel support vector machine. Progress In Electromagnetics Research, 2012. 130: pp. 369-388 
[13] Wu, J., Fruit classification by biogeography-based optimization and feedforward neural network. Expert Systems, 2016. 33(3): pp. 239-253

[14]Liu, G., Computer-aided diagnosis of abnormal breasts in mammogram images by weighted-type fractional Fourier transform. Advances in Mechanical Engineering, 2016. 8(2), Article ID: 11

[15]Chen, S., et al., Magnetic resonance brain image classification based on weighted-type fractional Fourier transform and nonparallel support vector machine. International Journal of Imaging Systems and Technology, 2015. 25(4): pp. 317-327

[16]Zhou, X.-X., Comparison of machine learning methods for stationary wavelet entropy-based multiple sclerosis detection: decision tree, k-nearest neighbors, and support vector machine. Simulation, 2016. 92(9): pp. 861-871

[17] Yang, M., Dual-Tree Complex Wavelet Transform and Twin Support Vector Machine for Pathological Brain Detection. Applied Sciences, 2016. 6(6), Article ID: 169

[18]Liu, G., Pathological brain detection in MRI scanning by wavelet packet Tsallis entropy and fuzzy support vector machine. SpringerPlus, 2015. 4(1), Article ID: 716

[19] Yang, J., Identification of green, Oolong and black teas in China via wavelet packet entropy and fuzzy support vector machine. Entropy, 2015. 17(10): pp. 6663-6682

[20] Liu, A., Magnetic resonance brain image classification via stationary wavelet transform and generalized eigenvalue proximal support vector machine. Journal of Medical Imaging and Health Informatics, 2015. 5(7): pp. 1395-1403

[21] Yang, J., Preclinical diagnosis of magnetic resonance (MR) brain images via discrete wavelet packet transform with Tsallis entropy and generalized eigenvalue proximal support vector machine (GEPSVM). Entropy, 2015. 17(4): pp. 1795-1813

[22] Wu, L., Weights optimization of neural network via improved BCO approach. Progress in Electromagnetics Research, 2008. 83: pp. 185-198

[23]Zhang, Y., Stock market prediction of S\&P 500 via combination of improved BCO approach and BP neural network. Expert systems with applications, 2009. 36(5): pp. 8849-8854

[24] Naggaz, N. and G. Wei, Remote-sensing Image Classification Based on an Improved Probabilistic Neural Network. Sensors, 2009. 9(9): pp. 7516-7539

[25] Wu, L., Crop Classification by forward neural network with adaptive chaotic particle swarm optimization. Sensors, 2011. 11(5): pp. 4721-4743

[26] Ji, G., Fruit classification using computer vision and feedforward neural network. Journal of Food Engineering, 2014. 143: pp. 167-177

[27]Feng, C., Feed-forward neural network optimized by hybridization of PSO and ABC for abnormal brain detection. International Journal of Imaging Systems and Technology, 2015. 25(2): pp. 153-164

[28] Sethy, P.K., et al. ANN BASED IMAGE RESTORATION IN APPROACH OF MULTILAYER PERCEPTRON. in International Conference on Inventive Computation Technologies (ICICT). 2016. Coimbatore, INDIA: IEEE. pp. 472-475

[29] Sun, Y., A Multilayer Perceptron Based Smart Pathological Brain Detection System by Fractional Fourier Entropy. Journal of Medical Systems, 2016. 40(7), Article ID: 173 
[30]30. Szczepanski, D. Multilayer perceptron for gait type classification based on inertial sensors data. in Federated Conference on Computer Science And Information Systems. 2016. Gdansk, POLAND: IEEE. pp. 947-950

[31]31. Ji, G., An MR brain images classifier system via particle swarm optimization and kernel support vector machine. The Scientific World Journal, 2013, Article ID: 130134

[32]32. Dong, Z., Classification of Alzheimer disease based on structural magnetic resonance imaging by kernel support vector machine decision tree. Progress In Electromagnetics Research, 2014. 144: pp. 171-184

[33]33. Zhou, X.-X., et al., Combination of stationary wavelet transform and kernel support vector machines for pathological brain detection. Simulation, 2016. 92(9): pp. 827-837

[34]34. Lu, S. and X. Qiu, A Pathological Brain Detection System based on Extreme Learning Machine Optimized by Bat Algorithm. CNS \& Neurological Disorders - Drug Targets, 2017. 16(1): pp. 23-29

[35]35. Lu, S. and Z. Lu A pathological brain detection system based on kernel based ELM. Multimedia Tools and Applications, 2016, DOI: 10.1007/s11042-016-3559-z (Online).

[36]36. Zhang, Y., et al., Genetic Pattern Search and Its Application to Brain Image Classification. Mathematical Problems in Engineering, 2013, Article ID: 580876

[37]37. Ji, G.L., A Rule-Based Model for Bankruptcy Prediction Based on an Improved Genetic Ant Colony Algorithm. Mathematical Problems in Engineering, 2013, Article ID: 753251

[38]38. Wu, L., UCAV path planning by fitness-scaling adaptive chaotic particle swarm optimization. Mathematical Problems in Engineering, 2013, Article ID: 705238

[39]39. Phillips, P., et al., Pathological brain detection in magnetic resonance imaging scanning by wavelet entropy and hybridization of biogeography-based optimization and particle swarm optimization. Progress In Electromagnetics Research, 2015. 152: pp. 41-58

[40]40. Ji, G., A comprehensive survey on particle swarm optimization algorithm and its applications. Mathematical Problems in Engineering, 2015, Article ID: 931256

[41]41. Yang, G., Automated classification of brain images using wavelet-energy and biogeography-based optimization. Multimedia Tools and Applications, 2016. 75(23): pp. 15601-15617

[42] 42. Wei, L., Fruit classification by wavelet-entropy and feedforward neural network trained by fitness-scaled chaotic ABC and biogeography-based optimization. Entropy, 2015. 17(8): pp. $5711-5728$

[43]43. Chen, P. and S. Du, Pathological Brain Detection via Wavelet Packet Tsallis Entropy and Real-Coded Biogeography-based Optimization. Fundamenta Informaticae, 2017. 151: pp. 275-291

[44]44. Abdelhakim, A.M., et al., A quality guaranteed robust image watermarking optimization with Artificial Bee Colony. Expert Systems with Applications, 2017. 72: pp. 317-326

[45]45. Wu, L., Optimal multi-level Thresholding based on Maximum Tsallis Entropy via an Artificial Bee Colony Approach. Entropy, 2011. 13(4): pp. 841-859

[46]46. Wu, L., Magnetic Resonance Brain Image Classification by an Improved Artificial Bee Colony Algorithm. Progress in Electromagnetics Research, 2011. 116: pp. 65-79 